Reprod. Nutr. Dévelop., 1982, 22 (5), 841-849.

\title{
Effet d'une carence protéique modérée sur la reproduction chez la rate et sur le développement des jeunes jusqu'au sevrage
}

\author{
B. BECK, J. M. DOLLET, J. P. MAX, G. DEBRY
}

\begin{abstract}
I.N.S.E.R.M. U. 59, Groupe de Recherches en Nutrition et Diététique, et

Département de Nutrition et des Maladies métaboliques de I'Université de Nancy I, 40, rue Lionnois, 54000 Nancy France.
\end{abstract}

Summary. Effect of moderate protein deficiency on reproduction in the female rat and on the development of the pups until weaning.

Two groups of Wistar female rats were respectively fed ad libitum a standard stock diet containing 22 p. 100 protein $(n=93)$ and a diet containing 7.5 p. 100 protein ( $n=189$ ) for 8 weeks. They were mated with male rats of the same strain after 2 weeks of these diets. A small decrease $(8 \mathrm{p}, 100)$ in fecundity was observed but this moderate protein deprivation did not affect either the litter size (9.68 \pm 3.50 vs $9.61 \pm 3.69$ ) or the percentage of stillborn pups (4.8 vs $4.9 \mathrm{p} .100$ ).

The postnatal mortality of the pups of deprived dams was much higher than that of pups from normal dams (11.2 vs 0.9 p. 100). During the suckling period, the $7.5 \mathrm{p} .100$ protein diet did not cover the requirements of the dams. They lost 20 p. 100 of their weight, whereas the weight of the dams fed the $22 \mathrm{p} .100$ protein diet remained stable. The weight deficit of the young rats born from deprived dams was about 10 p. 100 at birth but it rose to 50 p. 100 at weaning. During the gestation and suckling periods, the maternal body stores and tissues were mobilized to assure the growth of the young.

\section{Introduction.}

La carence protéino-énergétique a des effets très divers sur la fertilité, la gestation et la croissance chez l'animal et chez l'homme. Elle produit chez le rat une diminution de la ponte ovulaire et un accroissement de la résorption ou de I'élimination des œufs fécondés avant implantation (Gupta et Lacy, 1967). Les rates carencées ont également des portées réduites en nombre. Chez le chien, la maturité sexuelle est plus tardive et les périodes entre les ovulations sont plus longues (Heard et Stewart, 1971). Enfin pour toutes les espèces, la carence protéique se traduit par une baisse du poids des nouveau-nés (Susser, Marolla et Fleiss, 1972 ; Wittstruck et al., 1979 ; Massaro, Levitsky et Barnes, 1976 ; Platt, Heard et Stewart, 1964). 
Les avis divergent par contre au sujet de la relation existant pendant la gestation entre croissance fœtale, régimes ingérés par la mère et échanges entre mère et fœtus. Pour Rosso (1981 a) la taille et la vitalité du jeune rat sont uniquement influencées par la quantité et la qualité du régime ingéré par la mère alors que pour d'autres, la croissance fœtale peut être maintenue aux dépens même des propres réserves de la mère (in Hytten, 1964).

Le but de cette étude est double. Il s'agit d'une part de préciser l'effet de la carence protéique continue sur la gestation des rates et la croissance des ratons jusqu'au sevrage et d'autre part d'infirmer ou d'affirmer la notion de " parasitisme " de la mère par ses petits.

Le niveau protéique de la ration alimentaire utilisée est comparable à celui qui est observé dans les pays en voie de développement chez la femme en âge de procréer. Pour compléter le paraliélisme avec l'homme, les rats mâles sont soumis à la même carence protéique.

\section{Matériels et méthodes.}

Deux cent quatre-vingt-deux rates primipares $(188,9 \pm 9,6 \mathrm{~g})$ de souche Wistar (CESAL, Vigneul/Montmédy) sont séparées en deux groupes. Le groupe " contrôle " (n = 93) reçoit un régime commercial standard contenant 22 p. 100 de protéines (Régime AO3, UAR Villemoisson/Orge). Ce régime est utilisé comme référence car il permet d'obtenir une reproduction optimale chez le Rat. Le groupe " carencé » $(n=189)$ reçoit un régime contenant 7.5 p. 100 de protéines ( 9 p. 100 de caséine lactique). La composition complète des régimes est donnée dans le tableau 1. Les animaux vivent dans une animalerie climatisée (hygrométrie, température) avec une alternance jour-nuit de $12 \mathrm{~h}$, le jour débutant à $7 \mathrm{~h}$ le matin.

L'eau de boisson et la nourriture sont fournies ad libitum pendant les trois parties de l'expérience dont la durée totale est de huit semaines : 15 jours d'acclimatation aux régimes et à l'animalerie avant la mise au mâle, 3 semaines de gestation et 3 semaines après la mise-bas jusqu'au sevrage des petits. Dans chacun des deux groupes, 30 animaux servent de témoins pour les mesures de prise alimentaire : ils reçoivent les mêmes régimes que leurs homologues, mais une moitié (soit 15 animaux par groupe) n'est pas mise au mâle. Pendant la période d'acclimatation, les rats mâles $(198,3 \pm 7,8 \mathrm{~g})$ reçoivent le même régime que leurs homologues femelles.

Pour l'accouplement, la proportion d'un mâle pour trois femelles est respectée. Pendant les deux semaines qui suivent, les femelles sont gardées en cage plastique par groupe de 3 . Les femelles fécondées sont dénombrées puis placées en cage individuelle jusqu'à la fin de l'expérience.

Les rates sont pesées le jour de la mise au mâle, lors du changement de cages, après mise bas et au sevrage des ratons. Ceux-ci sont dénombrés et pesés dès leur naissance. Ils sont ensuite répartis à l'intérieur de chaque groupe à raison de 9 à 10 petits par mère. Ainsi les effets dus à la diminution du taux protéique des régimes ne sont pas accentués par l'existence de portées trop 
TABLEAU 1

Composition des régimes utilisés (en g pour $100 \mathrm{~g}$ )

Régime « contrôle ".

Protéines végétales (tourteau, levure)

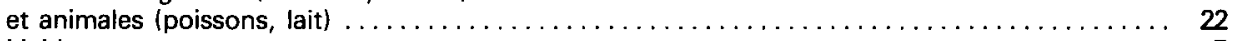

Lipides $\ldots \ldots \ldots \ldots \ldots \ldots \ldots \ldots \ldots \ldots \ldots \ldots \ldots \ldots \ldots \ldots \ldots \ldots \ldots \ldots \ldots \ldots \ldots \ldots \ldots \ldots \ldots \ldots 5$

Glucides $\ldots \ldots \ldots \ldots \ldots \ldots \ldots \ldots \ldots \ldots \ldots \ldots \ldots \ldots \ldots \ldots \ldots \ldots \ldots \ldots \ldots \ldots \ldots \ldots \ldots \ldots \ldots \ldots \ldots \ldots \ldots$

Cellulose $\ldots \ldots \ldots \ldots \ldots \ldots \ldots \ldots \ldots \ldots \ldots \ldots \ldots \ldots \ldots \ldots \ldots \ldots \ldots \ldots \ldots \ldots \ldots \ldots$

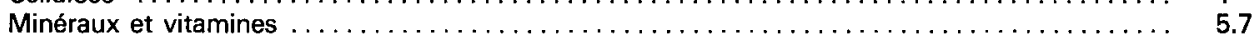

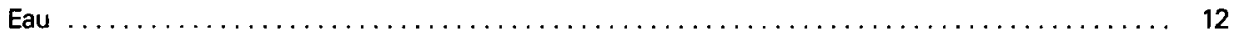

Régime « carence »

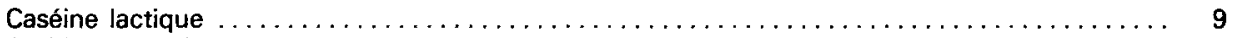

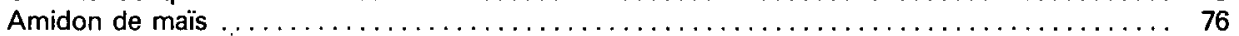

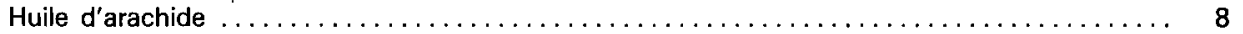

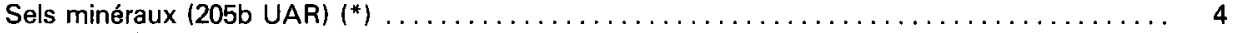

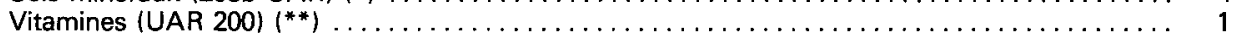

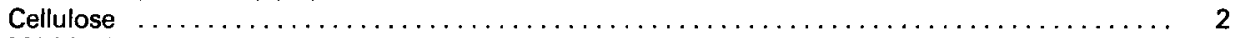

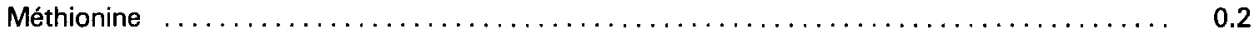

(*) Composition du mélange minéral (en g par $\mathrm{kg}$ de mélange) : $\mathrm{CaHPO}_{4}: 430 ; \mathrm{KCl}: 100$; $\mathrm{NaCl}: 100 ; \mathrm{MgCl}_{2}: 50 ; \mathrm{MgSO}_{4}: 50 ; \mathrm{Fe}_{2} \mathrm{O}_{3}: 3 ; \mathrm{FeSO}_{4} 7 \mathrm{H}_{2} \mathrm{O}: 5 ; \mathrm{MnSO}_{4} \mathrm{H}_{2} \mathrm{O}: 2.45 ; \mathrm{CuSO}$ $5 \mathrm{H}_{2} \mathrm{O}: 0.5 ; \mathrm{CoSO}_{4} 7 \mathrm{H}_{2} \mathrm{O}: 0.004 ; \mathrm{ZnSO}_{4} 7 \mathrm{H}_{2} \mathrm{O}: 2.008 ; \mathrm{KI}$ stabilisé : $0.008 ;$ OS : 1000 .

(**) Composition du mélange vitaminique (par $\mathrm{kg}$ de mélange) : A : 1980000 U.l.; $\mathrm{D}_{3}: 600000$ U.I. ; $\mathrm{B}_{1}: 2 \mathrm{~g} ; \mathrm{B}_{2}: 1,5 \mathrm{~g} ; \mathrm{B}_{3}: 7 \mathrm{~g} ; \mathrm{B}_{6} 1 \mathrm{~g} ; \mathrm{B}_{7}: 15 \mathrm{~g} ; \mathrm{B}_{12}: 0.005 \mathrm{~g} ; \mathrm{C}: 80 \mathrm{~g} ;$ $\mathrm{E}: 17 \mathrm{~g} ; \mathrm{K}_{3}: 4 \mathrm{~g}$; PP : $10 \mathrm{~g}$; Choline : $136 \mathrm{~g}$; Acide folique : $0,5 \mathrm{~g}$; Acide P.A.B. : $5 \mathrm{~g}$; Biotine $0.03 \mathrm{~g}$ QS : cellulose.

nombreuses. Au sevrage les petits sont à nouveau dénombrés et pesés. Les mesures de consommation alimentaire sont faites deux fois par semaine sur les lots témoins.

La méthode décrite ci-dessus dérive de celle préconisée par Libbin et Person (1979). Elle comporte en particulier un minimum de manipulations afin d'éviter tout stress générateur d'avortement précoce ou de cannibalisme maternel.

Les résultats sont exprimés en moyenne \pm écart-type. Ils sont comparés à l'aide du test $" t$ » de Student. $P<0,05$ est considéré comme significatif.

\section{Résultats.}

Les résultats concernant la fécondation des femelles et les différentes caractéristiques des portées ainsi que leur évolution sont regroupés dans le tableau 2. La taille moyenne des portées des mères carencées et le nombre des mort-nés ne sont pas significativement différents de ceux des mères " contrôle ". Par contre, dix fois plus de ratons nés de mères carencées sont éliminés pendant la période d'allaitement.

L'évolution du poids des femelles gravides et des femelles témoins est indiquée dans la figure 1. Le poids à l'accouplement de chaque lot n'est pas significativement différent. Au cours de toutes les étapes suivantes, le poids des femelles gravides du lot "contrôle " est significativement supérieur à celui des femelles gravides carencées ( $P<0,001 ; n=169$ ). A la fin de la période d'allaite- 


\section{TABLEAU 2}

Influence de la carence protéique modérée sur la fécondité et les caractéristiques des portées chez le rat

\begin{tabular}{lccccc}
\hline & $\begin{array}{c}\text { p. } 100 \\
\text { femelles } \\
\text { fécondées }\end{array}$ & $\begin{array}{c}\text { Nombre de } \\
\text { ratons par } \\
\text { portée }\left(^{*}\right)\end{array}$ & $\begin{array}{c}\text { p. } 100 \\
\text { mort-nés }\end{array}$ & $\begin{array}{c}\text { p. } 100 \\
\text { ratons } \\
\text { éliminés } \\
\text { au sevrage }\end{array}$ & $\begin{array}{c}\text { p. } 100 \\
\text { ratons } \\
\text { mâles } \\
\text { au sevrage }\end{array}$ \\
\hline $\begin{array}{l}\text { Régime contrôle }(22 \text { p. } 100 \\
\text { de protéines) } n=78 \ldots\end{array}$ & 73,1 & $9,68 \pm 3,50$ & 4,8 & 0,9 & 46,4 \\
\hline $\begin{array}{l}\text { Régime carencé }(7,5 \text { p. } 100 \\
\text { de protéines) } n=174 \ldots \ldots\end{array}$ & 65,5 & $9,61 \pm 3,69$ & 4,9 & 11,2 & 49,5 \\
\hline
\end{tabular}

(*) moyenne \pm écart type.

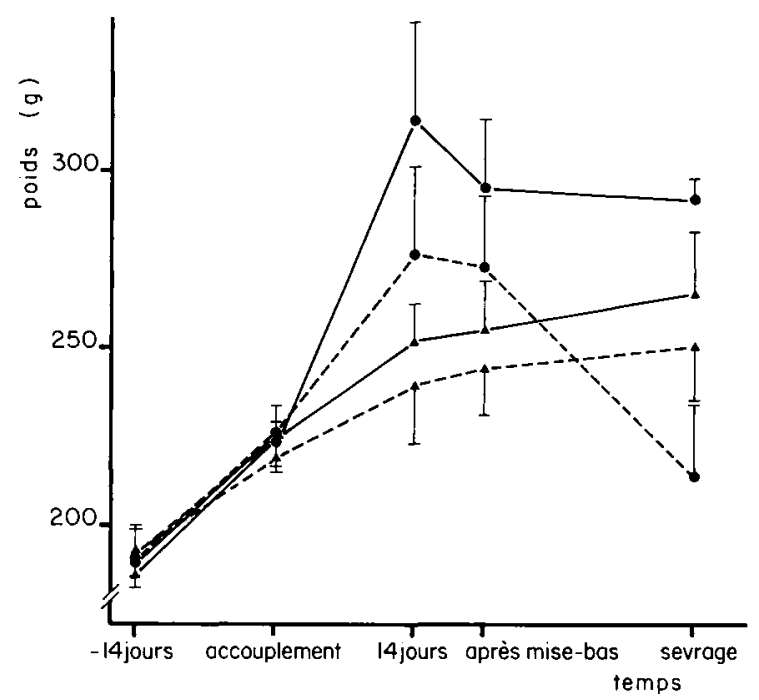

FIG. 1. - Evolution du poids (moyenne \pm écart type) des rates recevant respectivement un régime contenant 22 p. $100(-$ ou 7.5 p. $100(---)$ de protéines.

- : femelles gravides; $\boldsymbol{\Delta}$ : femelles témoins.

ment, le poids des rates gravides carencées est significativement inférieur à celui qui était le leur après la mise bas $(P<0,001 ; n=226)$. Cette variation n'existe pas dans le lot "contrôle ".

L'évolution du poids du couple mère-petit est indiquée dans la figure 2 .

Le poids de naissance des ratons de mères normales est significativement supérieur à celui des ratons de mères carencées $(7,07 \pm 1,13 \mathrm{~g}$ vs $6,36 \pm 0,97 \mathrm{~g}$ $-P<0,001 ; n=1569)$. II en est de même pour les poids des ratons au sevrage $(66,92 \pm 8,66 \mathrm{~g}$ vs $31,98 \pm 5,56 \mathrm{~g}-\mathrm{P}<0,001 ; \mathrm{n}=1446)$.

Les rates du lot témoin " carencé " non mises au mâle consomment environ 20 p. 100 de moins de nourriture que leurs homologues "contrôle " 
$(15,5 \pm 0,4$ vs $19,2 \pm 0,7 \mathrm{~g} / \mathrm{j})$. La gestation induit une augmentation de la prise alimentaire dans les deux groupes. Elle est plus importante dans le groupe de femelles gravides carencées $(+27,5$ p. 100$)$ que chez les femelles gravides " contrôle » $(+8,1$ p. 100), mais la quantité moyenne quotidienne absorbée par ces dernières reste quand même supérieure à celle de leurs homologues carencées $(+4,4$ p. 100).

Pendant la période d'allaitement, une nouvelle augmentation de la prise alimentaire se produit dans les deux groupes. La quantité moyenne de nourriture absorbée par les animaux est doublée pour le lot " carencé" " et plus que doublée pour le lot " contrôle ", atteignant respectivement $29,3 \pm 2,4$ et $50,4 \pm 7,0 \mathrm{~g} / \mathrm{j}$. La faible différence d'ingestion observée entre les régimes lors de la gestation $(4,4$ p. 100$)$ est presque décuplée $(41,90$ p. 100).

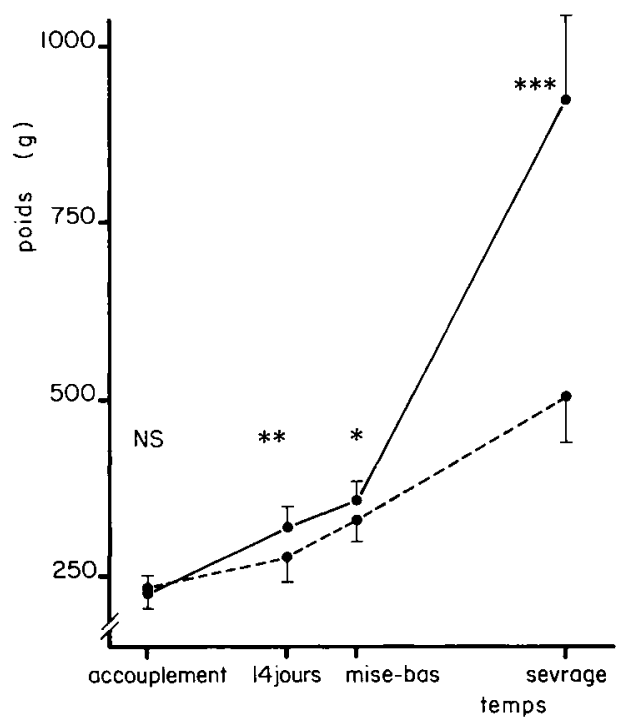

FIG. 2. - Evolution du poids (moyenne \pm écart type) du couple mère-petits recevant respectivement un régime contenant $22 \mathrm{p} .100(-)$ ou $7,5 \mathrm{p} .100(----)$ de protéines pendant les périodes de gestation et d'allaitement.

NS : non significatif ; ${ }^{*}: \mathrm{P}<0.05 ;{ }^{* *}: \mathrm{P}<0.01 ;{ }^{* * *}: \mathrm{P}<0.001$.

\section{Discussion.}

1) Effets sur la fécondité et la fertilité. - La carence protéique entraîne une faible diminution ( 8 p. 100 ) du nombre des femelles fertiles. Cette diminution peut être due à plusieurs phénomènes : troubles fœetaux in utero conduisant à l'avortement précoce, troubles de l'ovulation, résorption rapide des œufs chez la femelle, moindre fertilité du mâle ou anomalies des rapports mâle-femelle.

Les trois premières causes ont été jusqu'ici les plus discutées. Dans notre cas, elles ne semblent pas influencées par le taux protéique du régime puisque le 
nombre de petits à la naissance et le pourcentage des mort-nés ne sont pas différents entre le groupe " contrôle » et le groupe " carencé ". Les résultats, obtenus sur plus de 500 petits pour le régime à $22 \mathrm{p}$. 100 et plus de 1000 petits pour le régime à 7,5 p. 100 , sont en contradiction avec ceux obtenus par Gupta et Lacy (1967). Ces auteurs avaient observé une diminution du nombre d'œufs et du nombre de fœtus vivants après 2 semaines de gestation lorsque le régime comporte environ 10 p. 100 de protéines. Cette différence peut provenir d'une différence de qualité de protéines, car ils avaient utilisé le flocon d'avoine comme source principale de protides. Ils sont par contre en accord avec d'autres observations selon lesquelles une carence protéique modérée n'a qu'une très faible incidence sur la fécondité et la fertilité (Nelson et Evans, 1953; Bongaarts, 1980). Ceci est également vrai pour des carences protéiques très importantes chez certaines espèces domestiques polytoques telles que le porc (Pond et al., 1968 ; Leuillet, Etienne et Salmon-Legagneur, 1979).

Nos travaux indiquent que l'effet de la carence protéique suit la loi du tout ou rien : soit les femelles sont fécondées et mènent leur gestation à terme, soit elles ne sont pas fécondées. Ce défaut de fertilité peut être attribué aux causes hormonales (prolactine en particulier) invoquées par Callard et Leathem (1970) ou à des modifications des rapports mâle-femelle puisque la restriction protéique modérée $n^{\prime}$ affecte pas les performances reproductrices des rats mâles (Ghafoorunissa, 1980).

2) Effets sur l'état des petits et sur le comportement des mères. - Au point de vue morphologique, la seule différence notable concerne le poil des animaux : celui des ratons carencés est plus court, plus terne et nettement moins fourni que celui de leurs homologues " contrôle ". Par contre, l'apparition de la pilosité et l'ouverture des yeux se font dans les mêmes temps dans les deux groupes.

Bien que leur poids de naissance soit pratiquement normal, les ratons nés de mères carencées sont plus fragiles que ceux des mères "contrôle ", puisque leur taux de mortalité post-natale est plus que décuplé. Au sevrage, un important retard de croissance (50 p. 100 en poids corporel) est enregistré tout comme dans les travaux de Wittstruck et al. (1979) et El-Maraghi, Platt et Stewart (1966). Ce retard peut être attribué à une diminution de la quantité du lait produit par les mères (Mueller et Cox, 1946) en relation avec un sous développement des glandes mammaires (Rosso et al., 1981 b). La qualité du lait est également modifiée puisque les taux d'azote et de lipides sont respectivement diminués et augmentés (Crnic et Chase, 1978). Des résultats similaires ont été rapportés chez le porc (Pond et al., 1968 ; De Geeter et al., 1973).

Le taux élevé de mortalité postnatale traduit sans doute également un changement de comportement chez les mères. En effet, Frankova (1974), Weiner et al. (1977) et Gallo, Werboff et Knox (1980), ont observé un certain désintérêt des mères carencées pour leurs petits quand ils ne sont plus dans le nid. Ceux-ci dépérissent alors et les mères dénutries dévorent leurs petits morts ou trop faibles pour survivre.

3) Evolution pondérale des animaux. - L'évolution pondérale des femelles durant la gestation et l'allaitement permet d'apprécier les relations nutritionnelles 
existant entre les ratons et leur mère. Durant toute cette période, le couple mèreenfant forme une unité nutritionnelle et métabolique : la mère doit assurer sa propre croissance, l'acquisition de stocks énergétiques et la croissance de ses petits.

a) Pendant la gestation. - Durant la gestation, toutes les rates ont gagné du poids (poids après mise bas - poids à l'accouplement). Le gain de poids des rates gravides " contrôle " est beaucoup plus important que celui de leurs homologues carencées $(71,8$ vs 46,1$)$. Pendant la même période les rates non gravides ont une croissance normale, mais un peu plus rapide pour le lot à $22 \mathrm{p} .100$ $(28,7 \mathrm{~g}$ vs $23,4 \mathrm{~g})$.

Lederman et Rosso (1978) et Chow et Lee (1964) ont obtenu des résultats différents, l'administration d'un régime contenant 11 p. 100 des protéines ayant entraîné une faible perte de poids chez leurs rates. La faible qualité des protéines utilisées par ces derniers (blé, maïs et avoine principalement) intervient sans doute dans cette différence.

Cependant c'est le taux de protéines des régimes qui constitue l'élément majeur déterminant le gain pondéral maternel pendant la gestation (Lederman et Rosso, 1980). La diminution de ce taux à 7,5 p. 100 explique le déficit global constaté en fin de gestation. La stabilisation de ce déficit pendant la deuxième période de gestation (cf. fig. 2) indique une mobilisation des réserves corporelles des femelles carencées au profit de la croissance fœtale. Ces réserves sont moins importantes que celles de leurs homologues "contrôle " en raison d'un anabolisme diminué pendant la première phase de la gestation.

Pendant la gestation, seule la mère est vraiment touchée. Sa croissance est diminuée mais non stoppée et c'est elle qui assure les besoins du fœetus. Ce phénomène est d'autant plus net que les animaux carencés consomment moins que ceux qui reçoivent un régime optimal. Ces résultats sont proches de ceux obtenus par Anderson et al. (1980) chez des rates gravides dont l'apport protéique est réduit de 50 p. 100 soit pendant la phase anabolique, soit pendant la phase catabolique. Le rôle de " tampon » joué par les mères en cas de déficit nutritionnel est également reconnu chez d'autres espèces polytoques comme le porc chez qui les quantités de matière azotée fixées par les contenus utérins sont liées aux protéines ingérées (Leuillet, Etienne et Salmon-Legagneur, 1979).

b) Pendant la période d'allaitement. - Durant la lactation les besoins du couple mère-petit ne sont plus couverts par le régime à 7,5 p. 100 , et ceci malgré une augmentation conséquente de la prise alimentaire. (Déficit pondéral de plus de $400 \mathrm{~g}$ entre les deux lots. Cf fig. 2.) La mobilisation des réserves et des tissus maternels est beaucoup plus importante pendant cette période. Les rates carencées maigrissent de façon importante alors que les rates " contrôle " conservent un poids stable. Malgré cela, elles ne peuvent pas faire face aux besoins de leurs petits et au sevrage ceux-ci ont un poids inférieur de moitié à celui de leurs homologues non carencés.

Ainsi depuis la conception jusqu'au sevrage, les ratons "parasitent " leur mère. Pendant la gestation, leurs besoins sont encore faibles, aussi ne constatet-on qu'un défaut de croissance de la mère ; au contraire pendant la lactation, leurs besoins s'accroissent, contraignant la mère à cataboliser ses propres tissus. 
4) Relations avec les observations chez l'homme. - Un certain nombre de ces constatations peuvent être rapprochées de ce que l'on observe chez l'homme. Comme chez la rate gravide, la morbidité et la mortalité périnatale des enfants de mère carencée est accrue (Susser, Marolla et Fleiss, 1972). Comme dans notre expérience, Naeye et Tafari (1979) ont décrit une différence de poids très minime ( 7 p. 100$)$ entre les enfants de mères carencées et les enfants normaux. Quant au "parasitisme " il est bien connu chez la femme enceinte (Platt et Stewart, 1967).

\section{Conclusion.}

Les effets de la carence protéique précoce retentissent différemment sur les deux phases de la reproduction : pendant la gestation, seule la mère est vraiment touchée alors que pendant la période d'allaitement, les effets observés chez la mère se reportent massivement sur les petits. L'utilisation d'animaux en croissance permet dans ce cas de mieux apprécier le sens du flux des aliments vers les petits.

Reçu en août 1981.

Accepté en mai 1982.

Remerciements. - Les auteurs remercient Mr Antoine Fillion pour sa collaboration technique au cours de cette étude.

\section{Références}

ANDERSON G. D., AHOKAS R. A., LIPSHITZ J., DILTS P. B. Jr., 1980. Effect of maternal dietary restriction during pregnancy on maternal weight gain and fetal birth weight in the rat. J. Nutr., 110, 883-890.

BONGAARTS J., 1980. Does malnutrition affect fecundity? A summary of evidence. Science, 208, 564-569.

CALLARD I. P., LEATHEM J. H., 1970. Pregnancy maintenance in protein deficient rats. Acta endocrinol., 63, 539-544.

CHOW B. F., LEE C. J., 1964. Effect of dietary restriction of pregnant rats on body weight gain of the offspring. J. Nutr., 82, 10-18.

CRNIC L. S., CHASE H. P., 1978. Models of infantile undernutrition in rats. Effects on milk. J. Nutr., 108, 1755-1760.

DE Geeter M. J., hAYS V. W., KRATZer D. D., CROMWELL G. L., 1973. Potsweaning performance of pigs from gilts fed diets high and low in protein during gestation and lactation. J. anim. Sci., 37, 459-462.

EL-MARAGHI N. H. R., PLATT B. S., STEWART R. J. C., 1966. The effect of reproduction on the interaction of dietary protein and calcium. Br. J. Nutr., 20, 733-745.

FRANKOVA S., 1974. Effect of protein deficiency in early life and during lactation on maternal behavior. Baroda J. Nutr., 1, 1-24.

GALLO P. V., WERBOFF J., KNOX K., 1980. Protein restriction during gestation and lactation. Development of attachment behavior in cats. Behav. neur. Biol., 29, 216-223.

GHAFOORUNISSA, 1980. Undernutrition and fertility of male rats. J. Reprod. Fert., 59, 317-320.

GUPTA S. R., LACY B., 1967. Effects of protein calorie deficiency on the reproductive performance of female rats. Ind. J. med. Res., 55, 904-910.

HEARD C. R. C., STEWART R. J. C., 1971. Protein-calorie deficiency and disorders of the endocrine glands. Hormones, 2, 40-64. 
HYTTEN F. E., 1964. Nutritional aspects of foetal growth, 59-65. In MILLS C. F., PASSMORE R., Proc. 6th int. Congr. Nutr., E. S. Livingstone, Edinburgh and London.

LEDERMAN S. A., ROSSO P., 1978. The effects of food restriction during pregnancy on maternal weight and body composition. Fed. Proc., 37, 491 (Abstr.).

LEDERMAN S. A., ROSSO P., 1980. Effects of protein and carbohydrate supplements on fetal and maternal weight and on body composition in food restricted rats. Am. J. clin. Nutr., 33. 1912-1916.

LEUILLET M., ETIENNE M., SALMON-LEGAGNEUR E., 1979. Conséquences d'une très forte restriction azotée à différentes périodes de la gestation de la truie sur le développement des fœtus. Ann. Biol. anim. Bioch. Biophys., 19, 217-223.

LIBBIN R. M., PERSON P., 1979. Neonatal rat surgery : avoiding maternal cannibalism. Science, 206. 66.

MASSARO T. F., LEVITSKY D. A., BARNES R. H., 1976. Protein malnutrition induced during gestation : its effect on pup development and maternal behavior. Deve/op. Psychobiol., 10. 339-345.

MUELLER A. J., COX W. M. Jr., 1946. The effect of changes in diet on the volume and composition of rat milk. J. Nutr., 31, 249-259.

NAEYE R., TAFARI N., 1979. Effect of long term maternal undernutrition on the human foetus. Ped. Res., 13, 392 (Abstr.).

NELSON M. M., EVANS H. M., 1953. Relation of dietary protein levels to reproduction in the rat. J. Nutr., 51, 71-84.

PLATT B. S., HEARD C. R. C., STEWART R. J. C., 1964. Experimental protein calorie deficiency, 446-514. In MUNRO H. N., ALLISON J. B., Mammalian protein metabolism, Vol. 2, Acad. Press, New York.

PLATT B. S., STEWART R. J. C., 1967. Nutrition and the foetus. Matern. Child Care, 3, 539-543.

POND W. G., WAGNER W. C., DUNN J. A., WALKER E. F. Jr., 1968. Reproduction and early postnatal growth of progeny in swine fed a protein free diet during gestation. J. Nutr., 94, 309-316.

ROSSO P., 1981 a. Nutrition and maternal fetal exchange. Am. J. clin. Nutr., 34 (suppl.), 744-755.

ROSSO P., KEYOU G., BASSI J. A., SLUSSER W. M., 1981 b. Effect of malnutrition during pregnancy on the development of the mammary glands of rats. J. Nutr., 111, 1937-1941.

SUSSER M., MAROLLA F. A., FLEISS, 1972. Birth, weight, fetal age and perinatal mortality. Am. J. Epidemiol., 96, 197-203.

WEINER S. G., FITZPATRICK K. M., LEVIN R., SMOTHERMAN W. P., LEVINE S., 1977. Alterations in the maternal behavior of rats rearing malnourished offspring. Develop. Psychobiol., 10, 243-254.

WITTSTRUCK T. A., WOODWARD B. E., FORBES W. B., CASPI E., 1979. Influence of prenatal and continuous postnatal protein deficient diets on the development of rats. Biol. Neonate, 35, 1-7. 